A. Brancolini · G. Buttazzo · F. Santambrogio

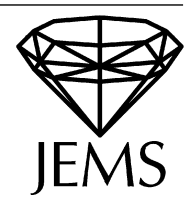

\title{
Path functionals over Wasserstein spaces
}

Received June 15, 2004

\begin{abstract}
Given a metric space $X$ we consider a general class of functionals which measure the cost of a path in $X$ joining two given points $x_{0}$ and $x_{1}$, providing abstract existence results for optimal paths. The results are then applied to the case when $X$ is a Wasserstein space of probabilities on a given set $\Omega$ and the cost of a path depends on the value of classical functionals over measures. Conditions for linking arbitrary extremal measures $\mu_{0}$ and $\mu_{1}$ by means of finite cost paths are given.
\end{abstract}

Keywords. Wasserstein distances, geodesics, irrigation trees, local functionals on measures

\section{Introduction}

Finding the best way to carry a given source to a given target is a problem that received a lot of attention in recent years. The literature on Monge-Kantorovich mass transportation problems and on Wasserstein distances is very rich, as well as on several related applications which include the shape optimization of elastic bodies (see for instance [7]), the design of public transportation networks (see for instance [9], [10], and [12]), the optimal location of production centres (see [8] for the asymptotics of the problem) and the study of irrigation trees (see for instance [3] and [14]).

The problem of transporting a source mass distribution to a target mass distribution by keeping together as much mass as possible during the transport, from which tree-shaped configurations arise, has been extensively studied, for instance in [3], [14], and [18]. In our new approach to this problem probability measures valued curves are considered, while the condition of keeping masses together is achieved by considering only measures supported in discrete sets.

Given a source or initial probability measure $\mu_{0}$ and a target or final probability measure $\mu_{1}$ we look for a path $\gamma$ in a Wasserstein space $\mathcal{W}_{p}(\Omega)$ that connects $\mu_{0}$ to $\mu_{1}$ and minimizes a suitable cost functional $\mathcal{J}(\gamma)$. In the present paper we consider functionals

A. Brancolini and F. Santambrogio: Scuola Normale Superiore, Piazza dei Cavalieri 7, 56126 Pisa, Italy; e-mail: a.brancolini@sns.it, f.santambrogio@sns.it

G. Buttazzo: Dipartimento di Matematica, Università di Pisa, Largo Bruno Pontecorvo 5, 56127 Pisa, Italy; e-mail: buttazzo@dm.unipi.it

Mathematics Subject Classification (2000): 49J45, 90B10, 58E10 
of the form

$$
\mathcal{J}(\gamma)=\int_{0}^{1} J(\gamma(t))\left|\gamma^{\prime}\right|(t) d t
$$

where $\left|\gamma^{\prime}\right|$ is the metric derivative of $\gamma$ in the Wasserstein space $\mathcal{W}_{p}(\Omega)$ and $J$ is a lower semicontinuous functional defined on measures. Here $J$ may be easily seen as the coefficient of a degenerate "Riemannian metric" on the space $\mathcal{W}_{p}(\Omega)$.

We restrict our analysis to the case of $J$ being a local functional over measures, an important class of functionals extensively studied by Bouchitté and Buttazzo in [4]-[6]. These functionals are the key tool in our approach, and among them we can find both functionals which are finite only on concentrated measures (for an application of them see [11] and [16]) and functionals which are finite only on spread measures. In fact, a particular point of interest in our approach is the fact that also different kinds of "Riemannian distances" are allowed (for instance those which prefer spread measures) by a change of the functional $J$.

In particular, we consider here the two extreme cases, in which the functional $J$ is one of the following:

$$
G_{r}(\mu)=\left\{\begin{array}{ll}
\sum_{k \in \mathbb{N}}\left(a_{k}\right)^{r} & \text { if } \mu=\sum_{k \in \mathbb{N}} a_{k} \delta_{x_{k}} \\
+\infty & \text { otherwise }
\end{array} \quad(0 \leq r<1),\right.
$$

whose domain is the space of purely atomic measures, or

$$
F_{q}(\mu)=\left\{\begin{array}{ll}
\int_{\Omega}|u|^{q} d x & \text { if } \mu=u \cdot \mathcal{L}^{N} \\
+\infty & \text { otherwise }
\end{array} \quad(q>1),\right.
$$

whose domain is the space $L^{q}(\Omega)$. We denote respectively by $\mathcal{G}_{r}$ the functional in 1.1 with $J$ replaced by $G_{r}$ and by $\mathcal{F}_{q}$ the same functional with $J$ replaced by $F_{q}$.

The first case is the one in which we get a "Riemannian distance" on probabilities which makes paths passing through concentrated measures cheaper. The second case, on the contrary, allows only paths which lie on $L^{q}(\Omega)$.

In both cases we analyze the question of the existence of optimal paths $\gamma_{\text {opt }}$ giving a finite value to the functional. When the domain $\Omega \subset \mathbb{R}^{N}$ is compact we find for the first case:

- if $\mu_{0}$ and $\mu_{1}$ are atomic measures, then an optimal path $\gamma_{\text {opt }}$ providing a finite value to $\mathcal{G}_{r}$ always exists;

- if $r>1-1 / N$, then the same is true for any pair of measures;

- if $r \leq 1-1 / N$, then there are measures $\mu_{0}$ and $\mu_{1}$ such that every path connecting them has an infinite cost.

Similarly, for the second case we have:

- if $\mu_{0}$ and $\mu_{1}$ are in $L^{q}(\Omega)$, then an optimal path $\gamma_{\text {opt }}$ providing a finite value to $\mathcal{F}_{q}$ always exists; 
- if $q<1+1 / N$, then the same is true for any pair of measures;

- if $q \geq 1+1 / N$, then there are measures $\mu_{0}$ and $\mu_{1}$ such that every path connecting them has an infinite cost.

In Section 4 we also discuss the case of unbounded domains such as $\Omega=\mathbb{R}^{N}$.

The analysis of existence results as well as the definition of the cost functionals is made in Section 2 in an abstract metric spaces framework, which can be used for future generalizations and developments.

In relation to the other papers mentioned at the beginning of this introduction it is not difficult to see that the model we propose is different and in general provides different solutions. However, among the different features our model supplies we may cite its mathematical simplicity and the possibility of performing standard numerical computations. From the mathematical point of view, our model reminds the construction of Riemannian metrics, as we already pointed out, and in connection to this we obtain fairly easy existence results for optimal paths. As far as numerics is concerned, we stress the fact that, when discretizing the metric derivative, the cost functional becomes a weighted sum of Wasserstein distances among couples of atomic probability measures which can be evaluated by well known algorithms such as the simplex method.

Anyway, we think that a comparison with the results presented by Xia in [18] and by Maddalena, Morel and Solimini in [14] will be important for future investigations. For instance, for the model proposed in [14] conditions for linking two prescribed measures by a finite cost configuration have been studied in [13] (while here and in [18] only conditions for linking arbitrary measures are provided): we do not know if similar conditions can be achieved in our case.

\section{The metric framework}

In this section a generic metric space $X$ with distance $d$ is considered. Under the assumption that closed bounded subsets of $X$ are compact, we will prove an existence result (Theorem 2.1) for variational problems with functionals of the type

$$
\mathcal{J}(\gamma)=\int_{0}^{1} J(\gamma(t))\left|\gamma^{\prime}\right|(t) d t
$$

where $\gamma:[0,1] \rightarrow X$ ranges among all Lipschitz curves such that $\gamma(0)=x_{0}$ and $\gamma(1)=x_{1}$. We will refer to the value of $\mathcal{J}$ at $\gamma$ as the energy of $\gamma$. By $\left|\gamma^{\prime}\right|(t)$ we denote the metric derivative of $\gamma$ at the point $t \in(0,1)$, i.e.

$$
\left|\gamma^{\prime}\right|(t)=\lim _{s \rightarrow t} \frac{d(\gamma(s), \gamma(t))}{|s-t|} .
$$

As a consequence of the Rademacher Theorem it can be seen (see [2]) that for any Lipschitz curve the metric derivative exists at almost every point (with respect to Lebesgue measure). Another useful result is that the variation of $\gamma$ can be written in terms of the metric derivative in integral form:

$$
\operatorname{Var}(\gamma)=\int_{0}^{1}\left|\gamma^{\prime}\right|(t) d t .
$$


By this formula it follows easily that $\left|\gamma^{\prime}\right| \leq M$ if and only if $\gamma$ is $M$-Lipschitz, since when $s<t$,

$$
d(\gamma(t), \gamma(s)) \leq \operatorname{Var}(\gamma,[s, t])=\int_{s}^{t}\left|\gamma^{\prime}\right|(\tau) d \tau \leq M|t-s|,
$$

the converse implication being immediate.

Theorem 2.1. Let $X$ be a metric space such that any closed bounded subset of $X$ is compact, $J: X \rightarrow[0,+\infty]$ be a lower semicontinuous function and $x_{0}, x_{1}$ arbitrary points in $X$. Then the functional

$$
\mathcal{J}(\gamma)=\int_{0}^{1} J(\gamma(t))\left|\gamma^{\prime}\right|(t) d t
$$

achieves a minimum value among all Lipschitz curves $\gamma:[0,1] \rightarrow X$ such that $\gamma(0)=$ $x_{0}$ and $\gamma(1)=x_{1}$, provided the following two assumptions are satisfied:

(H1) there exists a curve $\gamma_{0}$ such that $\mathcal{J}\left(\gamma_{0}\right)<+\infty$;

$$
\int_{0}^{\infty} \inf _{B_{r}\left(x_{0}\right)} J d r=+\infty .
$$

The proof of Theorem 2.1 relies on the following reparametrization lemma whose proof can be found for example in [2].

Lemma 2.2. Let $\gamma \in \operatorname{Lip}([0,1], X)$ and let $L=\operatorname{Var}(\gamma)$ be its total variation. Then there exists a Lipschitz curve $\tilde{\gamma} \in \operatorname{Lip}([0, L], X)$ such that $\left|\tilde{\gamma}^{\prime}\right|=1$ almost everywhere in $[0, L]$ and $\tilde{\gamma}$ is a parametrization of $\gamma$.

Proof of Theorem 2.1. Let $\left\{\gamma_{n}\right\}_{n \in \mathbb{N}}$ be a minimizing sequence and set $L_{n}=\operatorname{Var}\left(\gamma_{n}\right)$. Then the sequence $\left\{\mathcal{J}\left(\gamma_{n}\right)\right\}_{n \in \mathbb{N}}$ is bounded by a finite number $M$. By Lemma 2.2 there exists a sequence of curves $\tilde{\gamma}_{n}:\left[0, L_{n}\right] \rightarrow X$ parametrized with unit velocity, reparametrizing the given curves. We have

$$
M \geq \mathcal{J}\left(\gamma_{n}\right)=\int_{0}^{L_{n}} J\left(\tilde{\gamma}_{n}(t)\right) d t \geq \int_{0}^{L_{n}}\left(\inf _{B_{t}\left(x_{0}\right)} J\right) d t .
$$

Then $\left\{L_{n}\right\}_{n \in \mathbb{N}}$ is bounded, since otherwise, by assumption (H2), the right hand side would be unbounded. We can reparametrize each curve $\gamma_{n}$ with constant speed $L_{n}$, thus obtaining a new sequence $\left\{\hat{\gamma}_{n}\right\}_{n \in \mathbb{N}}$ in $\operatorname{Lip}([0,1], X)$, which is still a minimizing sequence, thanks to the equality $\mathcal{J}\left(\gamma_{n}\right)=\mathcal{J}\left(\hat{\gamma}_{n}\right)$. Since $\left\{L_{n}\right\}_{n \in \mathbb{N}}$ is bounded, this new minimizing sequence is uniformly bounded and uniformly Lipschitz. By the Ascoli-Arzelà Theorem we can suppose that, up to a subsequence, $\hat{\gamma}_{n} \rightarrow \hat{\gamma}$ uniformly for some $L$-Lipschitz curve $\hat{\gamma}$ where we have taken $L=\liminf _{n} L_{n}$. By recalling the link between Lipschitz conditions and metric derivative we have

$$
\left|\hat{\gamma}^{\prime}\right|(t) \leq L \quad \text { for a.e. } t \in[0,1]
$$


Now by using the lower semicontinuity of the functional $J$, we obtain

$$
\begin{aligned}
\mathcal{J}(\hat{\gamma}) & =\int_{0}^{1} J(\hat{\gamma}(t))\left|\hat{\gamma}^{\prime}\right|(t) d t \leq L \int_{0}^{1} \liminf _{n \rightarrow \infty} J\left(\hat{\gamma}_{n}(t)\right) d t \\
& \leq \liminf _{n \rightarrow \infty} L_{n} \int_{0}^{1} J\left(\hat{\gamma}_{n}(t)\right) d t=\liminf _{n \rightarrow \infty} \mathcal{J}\left(\hat{\gamma}_{n}\right),
\end{aligned}
$$

that is, the lower semicontinuity of $\mathcal{J}$ on the sequence considered, which completes the proof.

Remark 2.3. Notice that the integral assumption (H2) is always true if $J \geq c$ for a suitable strictly positive constant. Moreover Theorem 2.1 still holds if condition (H2) is replaced by the weaker assumption that there exists a curve $\gamma_{0}$ such that

$$
\mathcal{J}\left(\gamma_{0}\right)<\int_{0}^{+\infty} \inf _{B\left(x_{0}, r\right)} J d r .
$$

We give a slightly refined version of Theorem 2.1. which will be useful in the last section. The goal here is to weaken the compactness assumption on bounded subsets of $X$.

Theorem 2.4. Let $\left(X, d, d^{\prime}\right)$ be a metric space endowed with two different distances such that:

(K1) $d^{\prime} \leq d$;

(K2) all d-bounded sets in $X$ are relatively compact with respect to $d^{\prime}$;

(K3) the mapping $d: X \times X \rightarrow \mathbb{R}^{+}$is a lower semicontinuous function with respect to the distance $d^{\prime} \times d^{\prime}$.

Let $J: X \rightarrow[0,+\infty]$ be lower semicontinuous with respect to $d^{\prime}$. Consider the functional defined on the set of d-Lipschitz curves $\gamma:[0,1] \rightarrow X$ by

$$
\mathcal{J}(\gamma)=\int_{0}^{1} J(\gamma(t))\left|\gamma^{\prime}\right|_{d}(t) d t,
$$

where $\left|\gamma^{\prime}\right|_{d}(t)$ stands for the metric derivative of $\gamma$ with respect to $d$. Then, under the hypotheses $(\mathrm{H} 1)$ and $(\mathrm{H} 2)$ of Theorem 2.1 (where $B_{r}\left(x_{0}\right)$ are in the sense of $\left.d\right)$, there exists a minimum for $\mathcal{J}$.

Proof. We can take a minimizing sequence $\left\{\gamma_{n}\right\}_{n}$ and, as in Theorem 2.1, reparametrize it to obtain a sequence $\left\{\hat{\gamma}_{n}\right\}_{n}$ in which every curve has constant speed $L_{n}$. Hypothesis (H2) gives us the boundedness of $L_{n}$. Hence the sequence $\left\{\hat{\gamma}_{n}\right\}_{n}$ consists of $d$-equicontinuous functions from $[0,1]$ to a $d$-bounded subset of $X$. If we endow $X$ with the distance $d^{\prime}$ we have an equicontinuous (thanks to assumption (K1)) sequence of functions whose images are contained in a compact set. Consequently, we can use the Ascoli-Arzelà Theorem to choose a subsequence (not relabeled) such that $\hat{\gamma}_{n} \rightarrow \gamma\left(d^{\prime}\right.$-uniformly) for a suitable curve $\gamma$. The lower semicontinuity of $J$ with respect to $d^{\prime}$ allows us to use the Fatou 
Lemma and shows that $\gamma$ minimizes $\mathcal{J}$, provided we can show that $\gamma$ is $d$-Lipschitz with a Lipschitz constant not exceeding $\liminf _{n} L_{n}$. To do this we use assumption (K3). Given two points $s, t$ we have in fact

$$
d(\gamma(s), \gamma(t)) \leq \liminf _{n} d\left(\hat{\gamma}_{n}(s), \hat{\gamma}_{n}(t)\right) \leq \liminf _{n} L_{n}|s-t|,
$$

which shows the required Lipschitz property.

\section{The case of Wasserstein spaces}

In this section we consider a compact metric space $\Omega$ equipped with a distance function $c$ and a positive finite non-atomic Borel measure $m$. We consider the $p$-Wasserstein metric space $\mathcal{W}_{p}(\Omega)$. This is the space of Borel probability measures $\mu$ on $\Omega$ with finite moment of order $p$ with respect to a point $x_{0}$ (recall that the finiteness of the $p$-moment with respect to a point implies its finiteness with respect to any other point):

$$
\int_{\Omega} c\left(x, x_{0}\right)^{p} d \mu<+\infty
$$

equipped with the $p$-Wasserstein distance

$$
w_{p}\left(\mu_{1}, \mu_{2}\right)=\inf \left(\int_{\Omega \times \Omega} c(x, y)^{p} \lambda(d x, d y)\right)^{1 / p},
$$

where the infimum is taken over all transport plans $\lambda$ between $\mu_{1}$ and $\mu_{2}$, that is, over all probability measures $\lambda$ on $\Omega \times \Omega$ whose marginals $\pi_{\#}^{+} \lambda$ and $\pi_{\#}^{-} \lambda$ coincide with $\mu_{1}$ and $\mu_{2}$, respectively.

Notice that, since the distance $c$ is bounded, the space $\mathcal{W}_{p}(\Omega)$ consists of all probability measures. We consider functions $J$ on $\mathcal{W}_{p}(\Omega)$ that can be represented in the following form:

$$
J(\mu)=\int_{\Omega} f\left(\frac{d \mu}{d m}\right) d m+\int_{\Omega \backslash A_{\mu}} f^{\infty}\left(\frac{d \mu^{s}}{d\left|\mu^{s}\right|}\right) d\left|\mu^{s}\right|+\int_{A_{\mu}} g(\mu(x)) d \#(x),
$$

where

- $d \mu / d m$ is the Radon-Nikodym derivative of $\mu$ with respect to $m$,

- $f: \mathbb{R} \rightarrow[0,+\infty]$ is convex, lower semicontinuous and proper (i.e. not identically $+\infty)$

- $\mu^{s}$ is the singular part of $\mu$ with respect to $m$ according to the Radon-Nikodym decomposition theorem;

- $f^{\infty}$ is the recession function

$$
f^{\infty}(s):=\lim _{t \rightarrow+\infty} \frac{f\left(s_{0}+t s\right)}{t}
$$

(the limit is independent of the choice of $s_{0}$ in the domain of $f$, i.e. the set of points where $f$ is finite), 
- $A_{\mu}$ is the set of atoms of $\mu$, i.e. the points such that $\mu(x):=\mu(\{x\})>0$,

- $g: \mathbb{R} \rightarrow[0,+\infty]$ is a lower semicontinuous subadditive function such that $g(0)=0$,

- \# is the counting measure.

Note that our functional can be written in a simpler form since in our case $d \mu^{s} / d\left|\mu^{s}\right|=1$ for $\left|\mu^{s}\right|$-a.e. $x$, as $\mu$ is a positive measure:

$$
J(\mu)=\int_{\Omega} f\left(\frac{d \mu}{d m}\right) d m+f^{\infty}(1)\left|\mu^{s}\right|\left(\Omega \backslash A_{\mu}\right)+\int_{A_{\mu}} g(\mu(x)) d \#(x) .
$$

By the results that can be found in [4] and [5], these functionals are lower semicontinuous for the weak-* convergence of measures (and represent all local functionals with this semicontinuity property) whenever

$$
g_{0}(s):=\sup _{t>0} \frac{g(s t)}{t}=f^{\infty}(s) .
$$

Theorem 3.1. Suppose that $f(s)>0$ for $s>0$ and $g(1)>0$. Then $J \geq c>0$. In particular, the functional $\mathcal{J}$ defined on the set of Lipschitz curves $\gamma:[0,1] \rightarrow \mathcal{W}_{p}(\Omega)$ with given starting and ending points achieves a minimum, provided that there exists a curve with finite cost.

Proof. Let us fix some notation. By $\mu^{a}$ we mean the absolutely continuous part of $\mu$ with respect to the measure $m$, and by $\mu^{s}, \mu^{\#}, \mu^{c}$ respectively the singular part, the atomic part and the singular diffused part of $\mu$. Then we have $\mu=\mu^{a}+\mu^{s}=\mu^{a}+\mu^{c}+\mu^{\#}$. Since $f$ is convex, by the Jensen inequality we have

$$
\int_{\Omega} f\left(\frac{d \mu}{d m}\right) d m \geq m(\Omega) f\left(\frac{1}{m(\Omega)} \int_{\Omega} \frac{d \mu}{d m} d m\right)=m(\Omega) f\left(\frac{\mu^{a}(\Omega)}{m(\Omega)}\right) .
$$

Since $\mu$ is a positive measure and $f^{\infty}$ is 1-homogeneous,

$$
\int_{\Omega \backslash A_{\mu}} f^{\infty}\left(\frac{d \mu^{s}}{d\left|\mu^{s}\right|}\right) d\left|\mu^{s}\right|=\left|\mu^{s}\right|\left(\Omega \backslash A_{\mu}\right) f^{\infty}(1)=m(\Omega) f^{\infty}\left(\frac{\mu^{c}(\Omega)}{m(\Omega)}\right) .
$$

Since $g$ is a subadditive function,

$$
\int_{A_{\mu}} g(\mu(x)) d \#(x)=\sum_{x \in A_{\mu}} g(\mu(x)) \geq g\left(\sum_{x \in A_{\mu}} \mu(x)\right)=g\left(\mu^{\#}(\Omega)\right) .
$$

The recession function $f^{\infty}$ satisfies

$$
f^{\infty}(x) \geq f(x+y)-f(y) \text { for all } x, y \in \mathbb{R},
$$

and so the sum of the first two terms, i.e. those given by (3.2) and (3.3), can be estimated from below by

$$
m(\Omega) f\left(\frac{\mu^{a}(\Omega)+\mu^{c}(\Omega)}{m(\Omega)}\right) .
$$


Therefore summing up (3.2)-3.4 we obtain

$$
J(\mu) \geq m(\Omega) f\left(\frac{\mu^{a}(\Omega)+\mu^{c}(\Omega)}{m(\Omega)}\right)+g\left(\mu^{\#}(\Omega)\right) .
$$

We set $a=\mu^{\#}(\Omega)$; it follows that $1-a=\mu^{a}(\Omega)+\mu^{c}(\Omega)$. Since the function $a \mapsto$ $m(\Omega) f((1-a) / m(\Omega))+g(a)$ is lower semicontinuous, it attains a minimum in the interval $[0,1]$. Thanks to our hypothesis this sum is always positive, and so we have

$$
\min _{0 \leq a \leq 1} m(\Omega) f\left(\frac{1-a}{m(\Omega)}\right)+g(a)=c>0,
$$

that is, $J(\mu) \geq c>0$.

We now study some special cases of the functional we defined above. In the rest of this section $\Omega$ will be a compact convex subset of $\mathbb{R}^{N}$ and the measure $m$ will be the Lebesgue measure $\mathcal{L}^{N}$ on it.

\subsection{First case: $f=+\infty, g(z)=|z|^{r}(0 \leq r<1)$}

In this case we will denote the functional $J$ by $G_{r}$ and the corresponding functional $\mathcal{J}$ on Lipschitz paths will be called $\mathcal{G}_{r}$. This is the case when $G_{r}$ is finite only on purely atomic measures.

We are now going to consider the question whether there exists a curve connecting two given measures keeping our functional finite. First we prove that if both the initial and final measures are atomic the answer is positive. Then we prove that for $r$ in a suitable subinterval of $[0,1]$ every measure can be connected to a Dirac mass, hence every measure can be connected to any other measure by a path of finite energy. Finally, we show that this is not possible in general for every $r \in[0,1]$.

Theorem 3.2. Let $\mu_{0}$ and $\mu_{1}$ be convex combinations of Dirac masses, i.e.,

$$
\mu_{0}=\sum_{k=1}^{m} a_{k} \delta_{x_{k}}, \quad \mu_{1}=\sum_{l=1}^{n} b_{l} \delta_{y_{l}}
$$

with $a_{k}, b_{l}>0, \sum_{k} a_{k}=\sum_{l} b_{l}=1$. Then there exists a Lipschitz curve $\gamma:[0,1] \rightarrow$ $\mathcal{W}_{p}(\Omega)$ such that $\gamma(0)=\mu_{0}, \gamma(1)=\mu_{1}$ and

$$
\mathcal{G}_{r}(\gamma)=\int_{0}^{1} G_{r}(\gamma(t))\left|\gamma^{\prime}\right|(t) d t<+\infty .
$$

Proof. It is sufficient to prove the theorem when $a_{1}=1$, i.e. $\mu_{0}=\delta_{x_{1}}$, since in the general case one connects the first measure $\mu_{0}$ to a Dirac mass supported at an arbitrary point, and then one connects that Dirac mass to the final measure $\mu_{1}$. If one can keep the functional finite in both steps, then the result is proved in the general case. 
We now prove that the curve $\gamma:[0,1] \rightarrow \mathcal{W}_{p}(\Omega)$ given by

$$
\gamma(t)=\sum_{l=1}^{n} b_{l} \delta_{x_{1}+t\left(y_{l}-x_{1}\right)}
$$

is $w_{p}$-Lipschitz and $\mathcal{G}_{r}(\gamma)<+\infty$. Let $t_{1}$ and $t_{2}$ be time instants such that $t_{1}<t_{2}$. Then the transport plan between $\sum_{l} b_{l} \delta_{x_{1}+t_{1}\left(y_{l}-x_{1}\right)}$ and $\sum_{l} b_{l} \delta_{x_{1}+t_{2}\left(y_{l}-x_{1}\right)}$ induced by the map $T\left(x_{1}+t_{1}\left(y_{l}-x_{1}\right)\right):=x_{1}+t_{2}\left(y_{l}-x_{1}\right)$ gives

$$
\begin{aligned}
w_{p}\left(\gamma\left(t_{2}\right), \gamma\left(t_{1}\right)\right) & \leq\left(\int_{\Omega}|x-T(x)|^{p} d \gamma\left(t_{1}\right)\right)^{1 / p}=\left(\sum_{l=1}^{n} b_{l}\left|t_{2}-t_{1}\right|^{p}\left|y_{l}-x_{1}\right|^{p}\right)^{1 / p} \\
& =\left|t_{2}-t_{1}\right|\left(\sum_{l=1}^{n} b_{l}\left|y_{l}-x_{1}\right|^{p}\right)^{1 / p} .
\end{aligned}
$$

Hence the metric derivative with the respect to the Wasserstein $p$-distance is given by

$$
\left|\gamma^{\prime}\right|(t) \leq\left(\sum_{l=1}^{n} b_{l}\left|y_{l}-x_{1}\right|^{p}\right)^{1 / p}=w_{p}\left(\mu_{0}, \mu_{1}\right)
$$

On the other hand, we have

$$
G_{r}(\mu)= \begin{cases}\sum_{x \in A_{\mu}}|\mu(x)|^{r} & \text { if } \mu^{a}=\mu^{c}=0 \\ +\infty & \text { otherwise }\end{cases}
$$

Then

$$
G_{r}(\gamma(t))= \begin{cases}1 & \text { if } t=0 \\ \sum_{l=1}^{n} b_{l}^{r} & \text { if } t>0\end{cases}
$$

Hence

$$
\mathcal{G}_{r}(\gamma)=\int_{0}^{1} G_{r}(\gamma(t))\left|\gamma^{\prime}\right|(t) d t \leq \sum_{l=1}^{n}\left|b_{l}\right|^{r}\left(\sum_{l=1}^{n} b_{l}\left|y_{l}-x_{1}\right|^{p}\right)^{1 / p}<+\infty
$$

Remark 3.3. By repeating the proof of Theorem 3.2 one finds that the statement still holds for infinite sums of Dirac masses (i.e. $m=n=+\infty)$ provided $G_{r}\left(\mu_{0}\right)$ and $G_{r}\left(\mu_{1}\right)$ are finite, that is, $\sum_{k} a_{k}^{r}<+\infty$ and $\sum_{l} b_{l}^{r}<+\infty$.

The proof of the next theorem is related to the one of Proposition 3.1 of [18].

Theorem 3.4. Let $1-1 / N<r \leq 1$. Then, given two arbitrary $\mu_{0}$ and $\mu_{1}$ in $\mathcal{W}_{p}(\Omega)$, there exists a curve joining them such that the functional $\mathcal{G}_{r}$ is finite. 
Proof. It is sufficient to prove that every measure can be joined to a Dirac mass at an arbitrary point. We first prove the statement for $\Omega=[0,1]^{N}$. The dyadic subdivision of order $k$ of $Q=[0,1]^{N}$ is given by the family of closed $N$-dimensional cubes $\left\{Q_{h}^{k}\right\}_{h \in I_{k}}$, where $I_{k}=\left\{1,2,3, \ldots, 2^{k}\right\}^{N}$, obtained by dividing each edge of $Q$ into $2^{k}$ pieces of equal length. We will refer to the elements of $\left\{Q_{h}^{k}\right\}_{h \in I_{k}}$ as $k$-cubes. To every Borel regular finite measure $\mu$ we associate the following sequence of measures:

$$
\mu_{k}=\sum_{h \in I_{k}} b_{h}^{k} \delta_{y_{h}}
$$

where $b_{h}=\mu\left(Q_{h}^{k}\right)$ and $y_{h}$ is the centre of $Q_{h}^{k}$. It is straightforward to see that $\mu_{k} \rightarrow^{*} \mu$ as $k \rightarrow+\infty$.

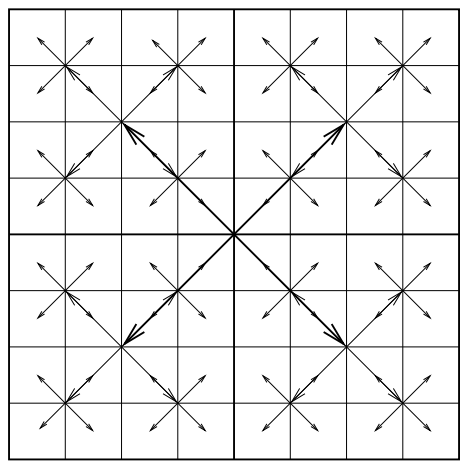

Fig. 1. Approximation at step $k=3$.

The idea is now simple (see Figure 11): first join $\mu_{k}$ to $\mu_{k+1}$ with an arc length parametrization $\gamma_{k}$, then put together all these curves to obtain a path from a Dirac mass to the measure $\mu$. At every step a $k$-cube is divided into $2^{N}$ parts which are $(k+1)$-cubes. To bring the Dirac mass at the centre of the $k$-cube to the $2^{N}$ centres of the $(k+1)$-cubes with the right weights at each centre one splits the centre of the $k$-cube into $2^{N}$ parts moving towards the centres of the adjacent $(k+1)$-cubes in such a way that each point moves with unit speed. At each step (see Figure 1 where the first three steps are represented) we obtain a curve $\gamma_{k}$ defined on an interval of length $(1 / 2)^{k} d / 2(d$ is the diagonal of $Q)$ such that $\left|\gamma_{k}^{\prime}\right|(t)=1$ for all $t$.

Let us now compute the value of the functional at the curve $\gamma$ made by joining all the curves $\gamma_{k}$ above. Since the function $f\left(x_{1}, \ldots, x_{n}\right)=\sum_{i=1}^{n} x_{i}^{r}$ with the constraint $\sum_{i=1}^{n} x_{i}=1$ reaches its maximum at the point $(1 / n, \ldots, 1 / n)$ we have

$$
\mathcal{G}_{r}(\gamma)=\sum_{k=1}^{\infty}\left(\frac{1}{2} \frac{1}{2^{k}} d \sum_{h \in I_{k}}\left(b_{h}^{k}\right)^{r}\right) \leq \sum_{k=1}^{\infty}\left(\frac{1}{2} \frac{1}{2^{k}} d 2^{N k}\left(\frac{1}{2^{N k}}\right)^{r}\right) .
$$

Since $1-1 / N<r \leq 1$ the sum considered above is convergent. 
In the case of a general $\Omega$ it is sufficient to consider a large cube containing the support of the measure $\mu$ such that the centre of the cube is in $\Omega$.

The bound given by $r>1-1 / N$ is sharp. We have in fact the following result.

Theorem 3.5. Suppose $r \leq 1-1 / N$. Then there exists a probability measure $\mu$ on $\Omega$ such that every non-constant $w_{p}$-Lipschitz path $\gamma$ such that $\gamma(0)=\mu$ has $\mathcal{G}_{r}(\gamma)=+\infty$.

Proof. Let $\Omega$ be the cube $[0,1]^{N}$ and $\mu$ the Lebesgue measure on it. We will show that

$$
\inf \left\{G_{r}(v) \mid w_{p}(\mu, v) \leq t\right\} \geq c t^{-N(1-r)} .
$$

Therefore, if $\gamma$ is a $w_{p}$-Lipschitz path with constant speed which starts from $\mu$, the integral defining $\mathcal{G}_{r}$ diverges. We can simply consider $t=2^{-k}$. To estimate $G_{r}(v)$ when $v$ is such that $w_{p}(\mu, v) \leq t$, consider a partition of $\Omega$ into small cubes of side $\varepsilon$. Let $k$ be the number of those cubes $Q_{i}$ such that $\nu\left(Q_{i}\right) \leq \mu\left(Q_{i}\right) / 2=\varepsilon^{N} / 2$. In all these cubes we have a zone in which the optimal transport map $s$ between $\mu$ and $v$ must take values outside the cube; this zone, given by $Q_{i} \backslash s^{-1}\left(Q_{i}\right)$, has a measure of at least $\varepsilon^{N} / 2$. We want to estimate from below the contribution of this zone to the total transport cost between $\mu$ and $v$. For this we may write

$$
\begin{aligned}
\int_{Q_{i} \backslash s^{-1}\left(Q_{i}\right)} d\left(x, \partial Q_{i}\right)^{p} d x & =\int_{0}^{(\varepsilon / 2)^{p}}\left|\left(Q_{i} \backslash s^{-1}\left(Q_{i}\right)\right) \cap\left\{d\left(x, \partial Q_{i}\right)^{p}>\tau\right\}\right| d \tau \\
& \geq \int_{0}^{(\varepsilon / 2)^{p}}\left(\frac{\varepsilon^{N}}{2}-\left|\left\{d\left(x, \partial Q_{i}\right)^{p} \leq \tau\right\}\right|\right) d \tau \\
& \geq \int_{0}^{B^{p} \varepsilon^{p}}\left(\frac{\varepsilon^{N}}{2}-\left|\left\{d\left(x, \partial Q_{i}\right) \leq B \varepsilon\right\}\right|\right) d \tau \geq c_{1} \varepsilon^{p} \varepsilon^{N}
\end{aligned}
$$

where $B$ is sufficiently small and $c_{1}$ is a positive constant. By recalling that the total transport cost (i.e. the $p$-th power of the distance $w_{p}$ ) is less than $t^{p}$, we have

$$
k c_{1} \varepsilon^{N+p} \leq t^{p}
$$

On the other hand, the value of $G_{r}$ can be estimated from below by means of the other cubes and we have

$$
G_{r}(v) \geq\left(\varepsilon^{-N}-k\right) c_{2} \varepsilon^{N r} .
$$

Let us now choose $\varepsilon=m t$ with $m$ an integer such that $c_{1} m^{p}>1$. By using 3.5, we have

$$
G_{r}(v) \geq t^{-N}\left(m^{-N}-m^{-N-p} / c_{1}\right) c_{2} m^{N r} t^{N r}=c_{3} t^{-N(1-r)},
$$

where the constant $c_{3}$ is positive.

For general $\Omega$ we can simply use a cube contained in $\Omega$ and show that the Lebesgue measure on it, rescaled to a probability measure, cannot be reached keeping the value of the integral finite. 
Example 3.6 (Y-shaped paths versus V-shaped paths). Consider the example in Figure2, where we suppose that $l$ and $h$ are fixed. For $0 \leq t \leq l_{0}$ we define

$$
x(t)=(t, 0)
$$

and for $l_{0} \leq t \leq l_{0}+\sqrt{l_{1}^{2}+h^{2}}$

$$
\begin{aligned}
& x_{1}(t)=\left(l_{0}+l_{1} \frac{t-l_{0}}{\sqrt{l_{1}^{2}+h^{2}}}, h \frac{t-l_{0}}{\sqrt{l_{1}^{2}+h^{2}}}\right), \\
& x_{2}(t)=\left(l_{0}+l_{1} \frac{t-l_{0}}{\sqrt{l_{1}^{2}+h^{2}}},-h \frac{t-l_{0}}{\sqrt{l_{1}^{2}+h^{2}}}\right) .
\end{aligned}
$$

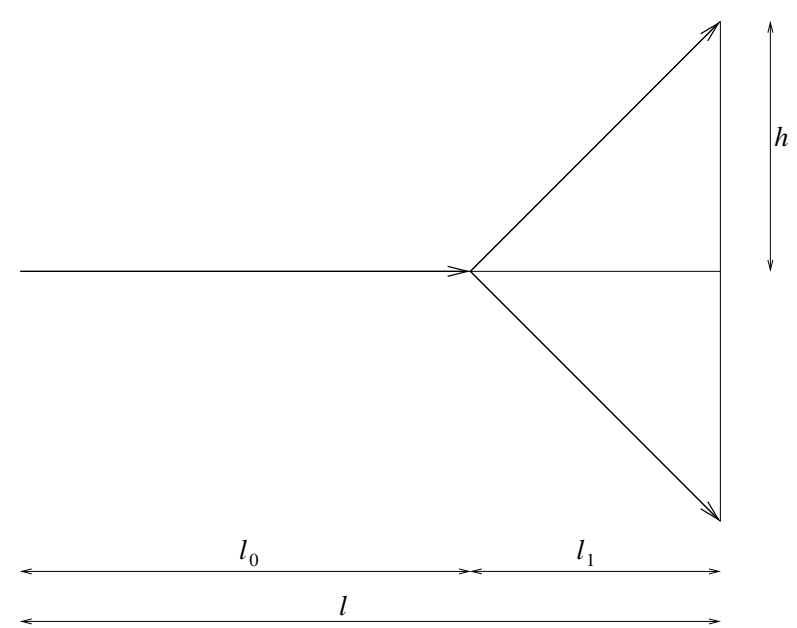

Fig. 2. A Y-shaped path for $r=1 / 2$.

Let us consider the curve $\gamma:\left[0, l_{0}+\sqrt{l_{1}^{2}+h^{2}}\right] \rightarrow \mathcal{W}_{p}(\Omega)$ defined by

$$
\gamma(t)= \begin{cases}\delta_{x(t)} & \text { if } 0 \leq t<l_{0} \\ \frac{1}{2} \delta_{x_{1}(t)}+\frac{1}{2} \delta_{x_{2}(t)} & \text { if } l_{0} \leq t \leq l_{0}+\sqrt{l_{1}^{2}+h^{2}}\end{cases}
$$

It is easy to see that $\left|\gamma^{\prime}\right|(t)=1$ and that

$$
\mathcal{G}_{r}(\gamma)=l_{0}+2^{1-r} \sqrt{\left(1-l_{0}\right)^{2}+h^{2}} .
$$

Then the minimum is achieved for

$$
l_{0}=l-\frac{h}{\sqrt{4^{1-r}-1}} .
$$

In particular, when $r=1 / 2$ we have a Y-shaped path (similar to the one of Figure 2) when $l>h$, while the path is $\mathrm{V}$-shaped when $l \leq h$. 
Remark 3.7. The result of Theorem 3.4 can clearly be improved for particular choices of $\mu_{0}$ and $\mu_{1}$. For instance, we can connect a Dirac mass to the $k$-dimensional Hausdorff measure on a smooth $k$-surface for all $r \in[1-1 / k, 1]$ (see also [14]).

\subsection{Second case: $f(z)=|z|^{q}(q>1), g=+\infty$}

We follow the same structure of the previous section. In this case we will denote the functional $J$ by $F_{q}$ and $\mathcal{J}$ by $\mathcal{F}_{q}$.

We start by proving that when $F_{q}\left(\mu_{0}\right)$ and $F_{q}\left(\mu_{1}\right)$ are finite, that is, $\mu_{0}$ and $\mu_{1}$ are measures with $L^{q}(\Omega)$ densities, the optimal path problem admits a solution with finite energy.

Theorem 3.8. Assume that $\mu_{0}=u_{0} \cdot \mathcal{L}^{N}, \mu_{1}=u_{1} \cdot \mathcal{L}^{N}$ with $u_{0}, u_{1} \in L^{q}(\Omega)$. Then $\mu_{0}$ and $\mu_{1}$ can be joined by a finite energy path.

The proof of this result relies on the notion of displacement convexity. This notion has been developed by McCann in [15] and is also described in [1] and [17].

Definition 3.9 (Displacement interpolation). Let $\mu_{0}$ and $\mu_{1}$ be two absolutely continuous probability measures on $\Omega$ and let $T: \Omega \rightarrow \Omega$ be an optimal transport map (unique if $p>1$ ) between $\mu_{0}$ and $\mu_{1}$ with respect to the cost function $|x-y|^{p}$. The $\operatorname{map} \gamma^{T}:[0,1] \rightarrow \mathcal{W}_{p}(\Omega)$ given by

$$
t \mapsto \gamma^{T}(t):=[(1-t) \operatorname{Id}+t T]_{\#} \mu_{0}
$$

is called $a$ displacement interpolation.

Remark 3.10. It is well known (see [1]) that the curve defined in $(3.6)$ is a geodesic in $\mathcal{W}_{p}(\Omega)$, parametrized in such a way that

$$
\left|\left(\gamma^{T}\right)^{\prime}\right|(t)=w_{p}\left(\mu_{0}, \mu_{1}\right) \quad \text { for a.e. } t .
$$

Definition 3.11 (Displacement convexity). A functional $F$ defined on all absolutely continuous measures (with respect to the Lebesgue measure) of $\mathcal{W}_{p}(\Omega)$ is said to be displacement convex if all the maps $t \mapsto F\left(\gamma^{T}(t)\right)$ are convex on $[0,1]$ for every choice of absolutely continuous measures $\mu_{0}, \mu_{1}$ and any optimal transport map $T$ as in Definition 3.9

The proof of the following criterion for displacement convexity can be found in [15] and [17] for the case $p=2$; a most complete one, dealing also with the case $p \neq 2$, is given in Proposition 9.3.9 of [1].

Theorem 3.12. Consider the following functional on the space $\mathcal{W}_{p}(\Omega)$, where $\Omega$ is any convex subset of $\mathbb{R}^{N}$ :

$$
F(\mu)= \begin{cases}\int_{\Omega} U\left(\frac{d \mu}{d \mathcal{L}^{N}}\right) d x & \text { if } \mu \text { is absolutely continuous } \\ +\infty & \text { otherwise }\end{cases}
$$


where $U:[0,+\infty] \rightarrow[0,+\infty]$ is a lower semicontinuous function with $U(0)=0$. If the map $r \mapsto r^{N} U\left(r^{-N}\right)$ is convex non-increasing on $] 0,+\infty[$, then the functional $F$ is displacement convex.

Remark 3.13. The assumption of $r \mapsto r^{N} U\left(r^{-N}\right)$ being convex and non-increasing easily implies that $U$ itself is convex.

Proof of Theorem 3.8 By Theorem 3.12 the functional $F_{q}$ is displacement convex, so that

$$
F_{q}\left(\gamma^{T}(t)\right) \leq(1-t) F_{q}\left(\mu_{0}\right)+t F_{q}\left(\mu_{1}\right)
$$

Then

$$
\begin{aligned}
\int_{0}^{1} F_{q}\left(\gamma^{T}(t)\right)\left|\left(\gamma^{T}\right)^{\prime}\right|(t) d t & \leq w_{p}\left(\mu_{0}, \mu_{1}\right) \int_{0}^{1}\left[(1-t) F_{q}\left(\mu_{0}\right)+t F_{q}\left(\mu_{1}\right)\right] d t \\
& =\frac{1}{2}\left(F_{q}\left(\mu_{0}\right)+F_{q}\left(\mu_{1}\right)\right) w_{p}\left(\mu_{0}, \mu_{1}\right) .
\end{aligned}
$$

Since $F_{q}\left(\mu_{0}\right)$ and $F_{q}\left(u_{1}\right)$ are finite, the path $t \mapsto \gamma^{T}(t)$ provides a finite value for the energy functional $\mathcal{F}_{q}$.

The next step will be the existence of an admissible path for arbitrary extremal measures, if $q$ satisfies some additional constraints.

Recall that if $\mu_{0}$ and $\mu_{1}$ are probability measures given by $L^{1}$ densities ( $u_{0}$ and $u_{1}$ respectively) and $T$ is a transport map between them with sufficient regularity, we have

$$
u_{1}(y)=u_{0}\left(T^{-1}(y)\right)\left|\operatorname{det} \mathrm{D} T^{-1}(y)\right| .
$$

Lemma 3.14. Let $q<1+1 / N$. Let also $\mu=u \cdot \mathcal{L}^{N}$ with $u \in L^{q}(\Omega)$ and $v=\sum_{j=1}^{k} b_{j} \delta_{y_{j}}$ with $\sum_{j=1}^{k} b_{j}=1$. Then there exists a path between $\mu$ and $v$ with finite energy.

Proof. Let $T$ be an optimal transport map between $\mu$ and $\nu$. Let $B_{j}:=T^{-1}\left(y_{j}\right)$. We now show that the path $\gamma^{T}$ has a finite energy. Set $T_{t}=(1-t) \operatorname{Id}+t T$. If $x \in B_{j}$, then $T_{t}(x)=(1-t) x+t y_{j}$ and $\operatorname{det} \mathrm{D} T_{t}(x)=(1-t)^{N}$. Let $u_{t}$ be the density of the measure $\left(T_{t}\right)_{\#} \mu$, that is,

$$
u_{t}(y)=u\left(T_{t}^{-1}(y)\right)\left|\operatorname{det} \mathrm{D} T_{t}^{-1}(y)\right| .
$$

We then have

$$
\begin{aligned}
\int\left|u_{t}(y)\right|^{q} d y & =\sum_{j=1}^{k} \int\left|u\left(\frac{y-t y_{j}}{1-t}\right)\right|^{q} \frac{1}{(1-t)^{N q}} d y \\
& =\sum_{j=1}^{k} \int|u(z)|^{q}(1-t)^{N(1-q)} d z=(1-t)^{N(1-q)} \int|u(z)|^{q} d z .
\end{aligned}
$$


Moreover, thanks to Remark 3.10, the metric derivative $\left|\gamma^{\prime}\right|(t)$ is constantly equal to the Wasserstein distance $w_{p}(\mu, v)$. Then

$$
\mathcal{F}_{q}(\gamma)=w_{p}(\mu, v) \int_{0}^{1} \int\left|u_{t}(y)\right|^{q} d y d t=\frac{w_{p}(\mu, \nu)}{N+1-N q} \int_{\Omega}|u|^{q} d x,
$$

which is finite since $q<1+1 / N$.

Theorem 3.15. Let $q<1+1 / N$. Then every couple of measures can be joined by a path with finite energy.

Proof. It is enough to link any measure $v$ to a fixed $L^{q}$ measure $\mu$ (for instance, the normalized Lebesgue measure) with a finite energy path. Let $\left\{v_{k}\right\}_{k \in \mathbb{N}}$ be a sequence of atomic measures approximating $v$ in the Wasserstein distance $w_{p}$. By Lemma 3.14, for every $k$ there is a path $\gamma_{k}$ with energy

$$
\mathcal{F}_{q}\left(\gamma_{k}\right)=C w_{p}\left(\mu, v_{k}\right)
$$

where $C$ is a constant which only depends on $N, q, \Omega$ (and of course $\mu$ ). Extracting a convergent subsequence of $\left\{\gamma_{k}\right\}_{k \in \mathbb{N}}$ provides a path $\gamma$ such that, by repeating the lower semicontinuity argument of Theorem 2.1.

$$
\mathcal{F}_{q}(\gamma) \leq \liminf _{k \rightarrow \infty} \mathcal{F}_{q}\left(\gamma_{k}\right)=\lim _{k \rightarrow \infty} C w_{p}\left(\mu, v_{k}\right)=C w_{p}(\mu, \nu) .
$$

Since $\gamma_{k}$ connects $\mu$ to $v_{k}$, it follows that $\gamma$ connects $\mu$ to $v$ and the result is established.

As in the previous section, we show that the previous result is sharp, as can be seen from the following statement which is valid in a more general setting. In fact, we prove an estimate which holds for every $w_{p}$-Lipschitz curve not only with values in $\mathcal{P}(\Omega)$, but also in $\mathcal{P}\left(\mathbb{R}^{N}\right)$.

Theorem 3.16. Suppose $q \geq 1+1 / N$. Then there exists $\mu \in \mathcal{W}_{p}(\Omega)$ such that every non-constant $w_{p}$-Lipschitz path $\gamma$ with $\gamma(0)=\mu$ has $\mathcal{F}_{q}(\gamma)=+\infty$.

Proof. Choose $\mu=\delta_{0}$ (supposing, up to translation, that $0 \in \Omega$ ). It is sufficient to prove that

$$
\inf \left\{F_{q}(v) \mid v \in \mathcal{P}(\Omega), w_{p}(\mu, v) \leq t\right\} \geq C t^{-N(q-1)},
$$

with $C>0$. In fact, by reparametrization, it is sufficient to prove that the functional is infinite on constant speed paths. Given such a path $\gamma$, with constant speed $L>0$, we have

$$
\mathcal{F}_{q}(\gamma)=L \int_{0}^{1} F_{q}(\gamma(t)) d t \geq L \int_{0}^{1} C(L t)^{-N(q-1)} d t=+\infty,
$$

where the integral diverges thanks to the assumption on $q$. To prove (3.7) we can suppose that $\Omega=\mathbb{R}^{N}$, which is the worst case. This shows that the result depends neither on the compactness nor on the convexity of $\Omega$. By considering the map that associates to every 
probability measure $\rho$ the measure $v=\left(m_{t}\right)_{\#} \rho$, where $m_{t}(x)=t x$, one has a one-toone correspondence between the probabilities whose Wasserstein distance from $\delta_{0}$ is less than 1 and those whose distance is less than $t$. It is easy to see that $v$ is $L^{q}$ if and only if the same happens for $\rho$ and that the density of $v$ is the function $x \mapsto t^{-N} u(x / t)$, where $u$ is the density of $\rho$. Therefore

$$
F_{q}(\nu)=\int \frac{u^{q}(x / t)}{t^{N q}} d x=\int u^{q}(y) t^{-N q} t^{N} d y=F_{q}(\rho) t^{-N(q-1)} .
$$

Consequently, it is now sufficient to evaluate the infimum in 3.7) when $t=1$, and this number will be the constant $C$ we are looking for. We will show that this infimum is in fact a minimum, thus proving that it is strictly positive. This problem is quite similar to those studied in [16]. To get the existence of a minimum we recall that the functional $F_{q}$ is sequentially lower semicontinuous with respect to the weak-* topology on probability measures, while the set $\left\{v \in \mathcal{P}\left(\mathbb{R}^{n}\right) \mid w_{p}\left(\delta_{0}, v\right) \leq 1\right\}$ is sequentially compact with respect to the same topology (in fact every sequence in it turns out to be tight).

Remark 3.17. As in the previous case, it is possible that two measures could be connected by a finite energy path even when $q>1+1 / N$. For instance, with $N=2$, the path given by

$$
\gamma(t)=\frac{1}{4 t} 1_{[-1,1] \times[-t, t]} \cdot \mathcal{L}^{2}
$$

is a Lipschitz path in $\mathcal{W}_{p}([-1,1] \times[-1,1])$ joining $\gamma_{0}=\frac{1}{2} \mathcal{H}^{1}\left\llcorner[-1,1]\right.$ to $\gamma_{1}=\frac{1}{4} \mathcal{L}^{2}$ (it is in fact a Wasserstein geodesic between them). The energy is finite as long as

$$
\int_{0}^{1} \frac{4 t}{(4 t)^{q}} d t<+\infty
$$

This condition is fullfilled when $1-q>-1$, i.e. when $q<2$, instead of the condition $q<1+1 / 2$ found in Theorem 3.15 .

\section{The case of unbounded domains}

The existence results of the previous section were based on two important facts: the compactness of the Wasserstein spaces $\mathcal{W}_{p}(\Omega)$ when $\Omega$ itself is compact and $1 \leq p<+\infty$, and the estimate like $F_{q} \geq c>0$, proven in Theorem 3.1, that can be obtained when $|\Omega|<+\infty$. Neither of these facts holds when $\Omega=\mathbb{R}^{N}$, for instance. This is the reason why we developed in Section 1 some tools giving the existence of optimal paths under weaker assumptions, even in the abstract metric setting. To replace the compactness of $\Omega$ we need to use Theorem 2.4. while to deal with the fact that we do not have $F_{q}(v) \geq c>0$ in the case where $v$ runs over all $\mathcal{W}_{p}\left(\mathbb{R}^{N}\right)$ we can use the weaker assumption given by hypothesis $(\mathrm{H} 2)$.

In this section we only deal with the case of $\mathcal{F}_{q}$-like functionals studied in the compact case in Section 3.2, the case of atomic measures and $\mathcal{G}_{r}$-like functionals of Section 3.1 still presents some extra difficulties when $\Omega$ is unbounded. We stress the fact that 
most of the techniques we use can be adapted to deal with several different cases, i.e. $\Omega$ unbounded but not necessarily the whole space, or the space $\mathcal{W}_{\infty}(\Omega)$ (where the distance is given by transport costs computed as a supremum instead of an integral). Notice that the use of Theorem 2.4 is necessary because in general, if $\Omega$ is not compact, the corresponding Wasserstein spaces are not even locally compact (and the same happens when we take $\Omega$ compact but we choose to consider the space $\mathcal{W}_{\infty}(\Omega)$ ), so we cannot have the compactness of closed balls.

First, we show some lemmas in order to use Theorem 2.4

Lemma 4.1. The weak topology (i.e. the one induced by the duality with the space $C_{b}(\Omega)$ of bounded continuous functions on $\Omega$ ) on the space $\mathcal{W}_{p}(\Omega)$ can be metrized by a distance $d^{\prime}$ such that $d^{\prime} \leq w_{1} \leq w_{p}$.

Proof. The usual distance metrizing the weak topology is given by

$$
d(\mu, v)=\sum_{k=1}^{\infty} 2^{-k}\left|\int \phi_{k} d(\mu-v)\right|
$$

where $\left(\phi_{k}\right)_{k}$ is a dense sequence in the unit ball of $C_{b}(\Omega)$. We can choose these functions to be Lipschitz continuous and let, for every index $k, c_{k}$ be the Lipschitz constant of $\phi_{k}$. Then

$$
d^{\prime}(\mu, v)=\sum_{k=1}^{\infty} \frac{2^{-k}}{1+c_{k}}\left|\int \phi_{k} d(\mu-v)\right|
$$

is a distance which metrizes the same topology. Since $\phi_{k} /\left(1+c_{k}\right)$ is a 1-Lipschitz function, thanks to the dual formulation of Monge's problem we have

$$
\left|\int \frac{\phi_{k}}{1+c_{k}} d(\mu-v)\right| \leq w_{1}(\mu, v),
$$

and so, by summing over $k$, we get $d^{\prime} \leq w_{1}$ as required.

The following two lemmas are well known.

Lemma 4.2. The distance $w_{p}$ is lower semicontinuous on $\mathcal{W}_{p}(\Omega) \times \mathcal{W}_{p}(\Omega)$ endowed with the weak $\times$ weak convergence.

Proof. Take $\mu_{n} \rightarrow \mu$ and $v_{n} \rightarrow v$. Let $\gamma_{n}$ be an optimal transport plan for the cost $|x-y|^{p}$ between $\mu_{n}$ and $\nu_{n}$; the sequence of these plans turns out to be tight thanks to tightness of the sequence of the marginal measures, and so we may suppose $\gamma_{n} \rightarrow \gamma$. We can now see that $\gamma$ is a transport plan between $\mu$ and $\nu$ and so

$w_{p}(\mu, v) \leq\left(\int|x-y|^{p} d \gamma\right)^{1 / p} \leq \liminf _{n \rightarrow+\infty}\left(\int|x-y|^{p} d \gamma_{n}\right)^{1 / p}=\liminf _{n \rightarrow \infty} w_{p}\left(\mu_{n}, v_{n}\right)$.

Lemma 4.3. All bounded sets in $\mathcal{W}_{p}\left(\mathbb{R}^{N}\right)$ are relatively compact with respect to the weak topology. 
Proof. Just notice that, in a bounded set, every sequence of probability measures turns out to be tight. The limits up to subsequences (that exist in the weak sense) still belong to the space $\mathcal{W}_{p}\left(\mathbb{R}^{N}\right)$ as a consequence of the lower semicontinuity of the functional $\mu \mapsto w_{p}\left(\mu, \delta_{0}\right)$ (which is nothing but the $p$-th moment of the measure).

We can now state our result.

Theorem 4.4. Let $F_{q}$ and $\mathcal{F}_{q}$ be defined as in Section 3.2 respectively on $\mathcal{W}_{p}\left(\mathbb{R}^{N}\right)$ and on the set of Lipschitz path in $\mathcal{W}_{p}\left(\mathbb{R}^{N}\right)$ joining two measures $\mu_{0}$ and $\mu_{1}$. Then

- if $q<1+1 / N$, for every $\mu_{0}$ and $\mu_{1}$ there exists a path giving finite and minimal value to $\mathcal{F}_{q}$;

- if $q \geq 1+1 / N$, there exist measures $\mu_{0}$ such that $\mathcal{F}_{q}=+\infty$ on every non-constant path starting from $\mu_{0}$.

Proof. Let us start from the case $q<1+1 / N$. Thanks to Lemmas 4.2 and 4.3 we can use Theorem 2.4 and so we just need to verify the two assumptions (H1) and $(\mathrm{H} 2)$. A finite energy path can be achieved in the same way as in Theorem 3.15, by passing through a fixed $L^{q}$ probability measure. Notice that, in order to have the convergence of a subsequence and the lower semicontinuity in the approximation by atomic measures, we will argue as in the proof of Theorem 2.4 instead of Theorem 2.1 In order to estimate the integral in $(\mathrm{H} 2)$ we will use the estimate given in Theorem 3.16 to obtain

$$
\inf \left\{F_{q}(v) \mid v \in \mathcal{P}(\Omega), w_{p}(\mu, v) \leq t\right\} \geq C t^{-N(q-1)},
$$

so that the integral diverges as long as $q<1+1 / N$.

By repeating the arguments of Theorem 3.16, we can then also prove the second part of our result, because $\mu=\delta_{0}$ cannot be joined to any other probability measure by a finite energy path.

Remark 4.5. In the previous theorem we did not mention the possibility to link, for arbitrary $q>1$, two measures $\mu_{0}, \mu_{1} \in L^{q}\left(\mathbb{R}^{N}\right)$. It is easy to check that the same construction used in Theorem 3.8 can also be used in this setting. We then get the existence of a path providing a finite value to $\mathcal{F}_{q}$, but some problems arise when we look for a minimal one. In fact for arbitrary $q$, condition $(\mathrm{H} 2)$ is no longer fulfilled and this prevents us from applying the general existence results.

To conclude this section, we highlight the difference between the case of the functional $\mathcal{F}_{q}$ we dealt with and the other important case, represented by the functional $\mathcal{G}_{r}$. In the latter case it is not necessary to pass through the divergence of the integral in assumption (H2), because we actually have $G_{r} \geq 1$, as already shown. On the other hand, some difficulties arise in verifying assumption (H1). In fact the construction we made to build a finite energy path linking $\delta_{0}$ to a probability measure $\mu$ strongly uses the compactness of the support of $\mu$. In order to get a similar construction for the case $\Omega=\mathbb{R}^{N}$ we would need an estimate like

$$
\inf \left\{w_{p}(\mu, v) \mid \# \operatorname{spt}(v) \leq k\right\} \leq C(\mu) k^{-1 / N},
$$


where $C(\mu)$ is a finite constant depending on the measure $\mu$. It is easy to get a similar estimate when $\mu$ has compact support, but the constant may depend on the diameter of the support. The existence of a similar estimate for arbitrary measures $\mu$ is linked to the asymptotics of the rescaled location problem in $\mathbb{R}^{N}$. A theory of this asymptotic problem has been explicitly developed (for instance in [8]) only in the case of compact support. However, it leads to a condition like $\mu^{N /(N+p)} \in L^{1}$, which is always fulfilled for $\mu$ compactly supported, while it may fail for general probability measures in $\mathcal{W}_{p}\left(\mathbb{R}^{N}\right)$. The existence of an estimate as above would easily imply a theorem similar to Theorem 4.4 for the case of $\mathcal{G}_{r}$, but we are not sure if such an estimate is necessary.

Acknowledgments. This work is part of the European Research Training Network "Homogenization and Multiple Scales"(HMS2000) under contract HPRN-2000-00109. The authors also acknowledge the support of the project "Problemi di Ottimizzazione in Teoria del Trasporto ed Applicazioni a Problemi di Pianificazione Urbana" of the Italian GNAMPA and of the project "Calcolo delle Variazioni" of the Italian Ministry of Education.

\section{References}

[1] Ambrosio, L., Gigli, N., Savarè, G.: Gradient Flows in Metric Spaces and in the Space of Probability Measures. Lectures Math. ETH Zürich 7, Birkhäuser, Basel (2005) Zbl pre02152346 MR 2129498

[2] Ambrosio, L., Tilli, P.: Selected Topics on "Analysis on Metric Spaces". Appunti dei Corsi Tenuti da Docenti della Scuola, Scuola Normale Superiore, Pisa (2000) Zbl pre02043480 MR 2012736

[3] Bernot, M., Caselles, V., Morel, J. M.: Are there infinite irrigation trees?. J. Math. Fluid Mech. 7 (2005)

[4] Bouchitté, G., Buttazzo, G.: New lower semicontinuous results for nonconvex functionals defined on measures. Nonlinear Anal. 15, 679-692 (1990) Zbl 0736.49007| MR 1073958

[5] Bouchitté, G., Buttazzo, G.: Integral representation of nonconvex functionals defined on Measures. Ann. Inst. H. Poincaré Anal. Non Linéaire 9, 101-117 (1992) Zbl 0757.49012 MR 1151468

[6] Bouchitté, G., Buttazzo, G.: Relaxation for a class of nonconvex functionals defined on measures. Ann. Inst. H. Poincaré Anal. Non Linéaire 10, 345-361 (1993) Zbl 0791.49016 MR 1230712

[7] Bouchitté, G., Buttazzo, G.: Characterization of optimal shapes and masses through MongeKantorovich equation. J. Eur. Math. Soc. (JEMS) 3, 139-168 (2001) Zbl 0982.49025 MR 1831873

[8] Bouchitté, G., Jimenez, C., Rajesh, M.: Asymptotique d'un problème de positionnement optimal. C. R. Acad. Sci. Paris 335, 853-858 (2002) Zbl 1041.90025 MR 1947712

[9] Brancolini, A., Buttazzo, G.: Optimal networks for mass transportation problems. ESAIM Control Optim. Calc. Var. 11, 88-101 (2005) Zbl pre05000004 MR 2110615

[10] Buttazzo, G., Oudet, E., Stepanov, E.: Optimal transportation problems with free Dirichlet regions. In: Variational Methods for Discontinuous Structures (Cernobbio, 2001), Progr. Nonlinear Differential Equations Appl. 51, Birkhäuser, 41-65 (2002) Zbl 1055.49029

[11] Buttazzo, G., Santambrogio, F.: A model for the optimal planning of an urban area. SIAM J. Math. Anal. 37, 514-530 (2005) MR 2176114 
[12] Buttazzo, G., Stepanov, E.: Optimal transportation networks as free Dirichlet regions for the Monge-Kantorovich problem. Ann. Scuola Norm. Sup. Pisa Cl. Sci. 2, 631-678 (2003) MR 2040639

[13] Devillanova, G., Solimini, S.: On the dimension of an irrigable measure. Preprint in preparation

[14] Maddalena, F., Morel, J. M., Solimini, S.: A variational model of irrigation patterns. Interfaces Free Bound. 5, 391-415 (2003) Zbl 1057.35076 MR 2031464

[15] McCann, R. J.: A convexity principle for interacting gases. Adv. Math. 128, 153-159 (1997) Zbl 0901.49012 MR 1451422

[16] Santambrogio, F.: Misure ottime per costi di trasporto e funzionali locali. Tesi di Laurea (2003); http://cvgmt.sns.it/

[17] Villani, C.: Topics in Optimal Transportation. Grad. Stud. in Math. 58, Amer. Math. Soc., Providence, RI (2003) Zbl pre01909499 MR 1964483

[18] Xia, Q.: Optimal paths related to transport problems. Commun. Contemp. Math. 5, 251-279 (2003) Zbl 1032.90003 MR 1966259 